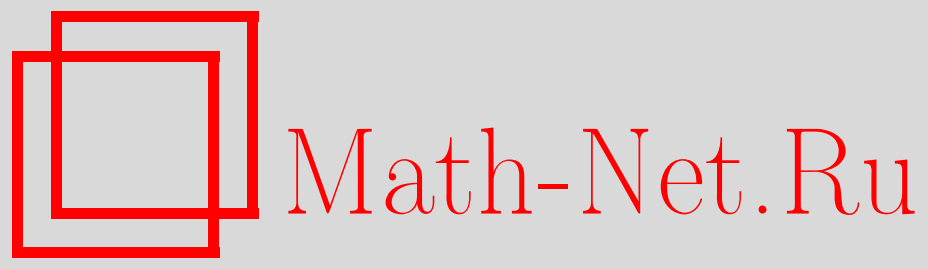

Р. С. Авдеев, Н. Е. Горфинкель, Гармонический анализ на сферических однородных пространствах с разрешимым стабилизатором, Функи. анализ и его прил., 2012, том 46, выпуск 3, 1-15

DOI: https://doi.org/10.4213/faa3080

Использование Общероссийского математического портала MathNet.Ru подразумевает, что вы прочитали и согласны с пользовательским соглашением

http://www . mathnet.ru/rus/agreement

Параметры загрузки:

IP : 54.197 .217 .227

26 апреля 2023 г., 13:48:03

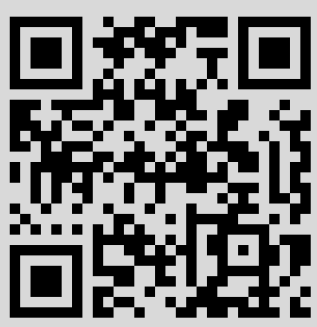


Функционалъный анализ и его приложения

2012, т. 46, вып. 3, с. 1-15

УДК 512.745+517.986.6

\title{
Гармонический анализ на сферических однородных пространствах с разрешимым стабилизатором*
}

\author{
(c) 2012. Р. С. АвДЕев, Н. Е. ГОРфИнкель
}

\begin{abstract}
Для всех сферических однородных пространств $G / H$, где $G$ - односвязная полупростая алгебраическая группа, а $H$ - ее связная разрешимая подгруппа, вычисляются спектры представлений группы $G$ в пространствах регулярных сечений однородных линейных расслоений над $G / H$.
\end{abstract}

\section{§1. Введение}

1.1. Пусть $G$ - связная полупростая комплексная алгебраическая группа и $H$ - ее замкнутая подгруппа. Одной из задач гармонического анализа на однородном пространстве $G / H$ является нахождение спектра представления группы $G$ в пространстве $\mathbb{C}[G / H]$ регулярных функций на $G / H$. Важной характеристикой этого спектра является так называемая полугруппа стариих весов $\Lambda_{+}(G / H)$. Она состоит из тех доминантных весов группы $G$, для которых пространство $\mathbb{C}[G / H]$, рассматриваемое как $G$-модуль, содержит неприводимый $G$-подмодуль со старшим весом $\lambda$.

Описанная выше задача допускает естественное обобщение. Таким обобщением является задача о нахождении спектров представлений группы $G$ в пространствах регулярных сечений однородных линейных расслоений над $G / H$. Совокупность этих спектров также определяет некоторую полугруппу $\widehat{\Lambda}_{+}(G / H)$, которая называется расширенной полугруппой стариих весов однородного пространства $G / H$. Точное определение этой полугруппы будет дано в разд. 1.2. Полугруппа $\Lambda_{+}(G / H)$ естественным образом отождествляется с подполугруппой в $\widehat{\Lambda}_{+}(G / H)$ (см. разд. 1.2$)$.

Подгруппа $H \subset G$ называется сферической (однородное пространство $G / H$ называется сферическим), если борелевская подгруппа $B \subset G$ имеет открытую орбиту в $G / H$. В работе [1] доказан следующий критерий сферичности подгруппы $H$.

Теорема 1 [1, теорема 1]. (1) Подгруппа $H \subset G$ является сферической тогда и только тогда, когда для всякого однородного линейного расслоения $L$ над $G / H$ спектр представления группљ $G$ в пространстве регулярных сечений расслоения $L$ прост.

(2) Если многообразие $G / H$ квазиафбинно, то подгруппа $H \subset G$ является сферической тогда и только тогда, когда спектр представления группъ $G$ в пространстве $\mathbb{C}[G / H]$ регулярных функиий на $G / H$ прост.

Из этой теоремы вытекает, что для сферического однородного пространства $G / H$ полугруппа $\widehat{\Lambda}_{+}(G / H)$ определяет $G$-модульную структуру в пространствах регулярных сечений всех однородных линейных расслоений над

*Работа выполнена при частичной финансовой поддержке РФФИ (грант 09-01-00648-а). 
$G / H$, а полугруппа $\Lambda_{+}(G / H)$ определяет структуру $G$-модуля в пространстве $\mathbb{C}[G / H]$. В связи с этим представляет интерес вычисление полугрупп $\Lambda_{+}(G / H)$ и $\widehat{\Lambda}_{+}(G / H)$ для сферических однородных пространств $G / H$.

На настоящий момент значительные продвижения достигнуты в вычислении полугрупп старших весов для аффинных сферических однородных пространств $G / H$ (т.е. таких, для которых $H$ редуктивна). А именно, имеется описание полугрупп $\Lambda_{+}(G / H)$ для всех строго неприводимых односвязных аффинных сферических однородных пространств $G / H$ (их определение см., например, в $[2$, п. 1.4]), к числу которых относятся все односвязные аффинные сферические однородные пространства простых групп. Для таких пространств $G / H$ в случае простой группы $G$ в работе [3] вычислены полугруппы $\Lambda_{+}(G / H)$, а в случае непростой $G$ в работе [2] вычислены полугруппы $\widehat{\Lambda}_{+}(G / H)$. Используя эти результаты, с учетом несложных дополнительных соображений можно получить описание полугрупп $\widehat{\Lambda}_{+}(G / H)$ для всех односвязных аффинных сферических однородных пространств $G / H$, однако этот момент пока не отражен в литературе.

Замечание 1. Насколько известно авторам настоящей работы, в случае непростой группы $G$ полугруппы $\widehat{\Lambda}_{+}(G / H)$ для всех односвязных строго неприводимых аффинных сферических однородных пространств $G / H$ были вычислены Ю. В. Дзядыком еще в 1985 г. Однако эти результаты до сих пор не опубликованы.

В случае неаффинных сферических однородных пространств $G / H$ ситуация с вычислением полугрупп $\Lambda_{+}(G / H)$ или $\widehat{\Lambda}_{+}(G / H)$ значительно сложнее: авторам настоящей работы известны только некоторые частные случаи вычисления этих полугрупп. Например, полугруппу $\widehat{\Lambda}_{+}(G / H)$ несложно описать для случая, когда $H$ является промежуточной подгруппой между некоторой параболической подгруппой группы $G$ и ее коммутантом (такие подгруппы $H-$ это в точности орисферические подгруппы, т. е. подгруппы, содержащие некоторую максимальную унипотентную подгруппу группы $G$; об этом см. [4, п. 2]). Другой частный случай рассмотрен в [5], где вычислены полугруппы $\widehat{\Lambda}_{+}(G / H)$ в ситуации, когда группа $G$ односвязна и $H=T U^{\prime}(U-$ максимальная унипотентная подгруппа в $G, U^{\prime}-$ ее коммутант, $T$ - максимальный тор в $G$, нормализующий $U)$.

В общем случае для сферических однородных пространств $G / H$ представляется более удобным вычислять полугруппу $\widehat{\Lambda}_{+}(G / H)$. Это обусловлено следующей причиной: если группа $G$ односвязна, то для любой сферической подгруппы $H \subset G$ полугруппа $\widehat{\Lambda}_{+}(G / H)$ свободна (см. теорему 2 в разд. 1.3$)$. В этом случае для ее вычисления достаточно найти ее ранг и предъявить в нужном количестве ее неразложимые элементы. Что касается полугруппы $\Lambda_{+}(G / H)$, то она оказывается свободной гораздо реже. А именно, в случае односвязной $G$ и связной $H$ известным достаточным условием свободности этой полугруппы является отсутствие у группы $H$ нетривиальных характеров (см. [6, предложение 2]). Отметим, что, вообще говоря, это условие является весьма ограничительным.

В настоящей работе вычисляются полугруппы $\widehat{\Lambda}_{+}(G / H)$ для всех сферических однородных пространств $G / H$, где $G$ - односвязная полупростая группа и $H$ - ее связная разрешимая подгруппа. Используемый для этой цели подход 
соединяет в себе идеи, содержащиеся в работах [5] и [7]. А именно, метод вычисления полугрупп $\widehat{\Lambda}_{+}(G / H)$ в случае $H=T U^{\prime}$ (см. выше), применявшийся в [5], мы обобщаем на случай произвольной связной разрешимой сферической подгруппы $H \subset G$, используя при этом развитую в [7] структурную теорию связных разрешимых сферических подгрупп в полупростых алгебраических группах. Результаты вычислений формулируются в терминах тех комбинаторных данных, которые рассматриваются в [7]. Основным результатом работы является теорема 4 (см. разд. 2.1).

1.2. В данной работе основным полем является поле $\mathbb{C}$ комплексных чисел, все топологические термины относятся к топологии Зарисского, все рассматриваемые группы предполагаются алгебраическими, а их подгруппы - замкнутыми. Касательные алгебры групп, обозначенных прописными латинскими буквами, обозначаются соответствующими строчными готическими буквами. Для любой группы $L$ через $\mathfrak{X}(L)$ обозначается группа ее характеров, записываемая аддитивно.

Везде далее через $G$ обозначается связная полупростая алгебраическая группа. Предполагается, что в $G$ фиксированы борелевская подгруппа $B$ и содержащийся в ней максимальный тор $T$. Буквой $U$ обозначается максимальная унипотентная подгруппа группы $G$, содержащаяся в $B$. Группа $\mathfrak{X}(B)$ отождествляется с $\mathfrak{X}(T)$ посредством ограничения характеров с $B$ на $T$. Множество доминантных весов группы $G$ по отношению к $B$ обозначается через $\Lambda_{+}(G)$, $\Lambda_{+}(G) \subset \mathfrak{X}(B)$. Это обозначение согласуется с введенным в разд. 1.1 обозначением $\Lambda_{+}(G / H)$ в случае $H=\{e\}$. При $\lambda \in \Lambda_{+}(G)$ через $V(\lambda)$ обозначается неприводимый $G$-модуль со старшим весом $\lambda$, а через $v_{\lambda}$ - фиксированный старший вектор в $V(\lambda)$ (по отношению к $B$ ). Для всякого веса $\lambda \in \Lambda_{+}(G)$ через $\lambda^{*}$ обозначается старший вес неприводимого $G$-модуля, сопряженного к $V(\lambda)$.

Действия группы $G$ самой на себе левыми сдвигами $((g, x) \mapsto g x)$ и правыми сдвигами $\left((g, x) \mapsto x g^{-1}\right)$ индуцируют ее представления в пространстве $\mathbb{C}[G]$ регулярных функций на $G$ по формулам $(g f)(x)=f\left(g^{-1} x\right)$ и $(g f)(x)=f(x g)$ соответственно $(g, x \in G, f \in \mathbb{C}[G])$. Для краткости первое из этих действий будем называть действием слева, а второе - действием справа. Для любой подгруппы $L \subset G$ через ${ }^{L} \mathbb{C}[G]$ (соответственно $\mathbb{C}[G]^{L}$ ) будем обозначать алгебру функций из $\mathbb{C}[G]$, инвариантных относительно действия $L$ слева (соответственно справа).

Пусть $H \subset G$ - произвольная подгруппа. Напомним (см. [8, теорема 4]), что однородные линейные расслоения над $G / H$ находятся во взаимно однозначном соответствии с характерами группы $H$. А именно, характеру $\chi \in \mathfrak{X}(H)$ отвечает однородное линейное расслоение $L(\chi)=\left(G \times \mathbb{C}_{\chi}\right) / H$ над $G / H$, где $H$ действует на $G$ правыми сдвигами, а в пространстве $\mathbb{C}_{\chi} \simeq \mathbb{C}$ - при помощи характера $\chi$. Слоем расслоения $L(\chi)$ над точкой $e H$ является прямая $\mathbb{C}_{\chi}$. При каждом $\chi \in \mathfrak{X}(H)$ имеется естественный $G$-эквивариантный изоморфизм между пространством $\Gamma(L(-\chi))$ регулярных сечений расслоения $L(-\chi)$ и пространством

$$
V_{\chi}=\{f \in \mathbb{C}[G] \mid f(g h)=\chi(h) f(g) \forall g \in G, \forall h \in H\} \subset \mathbb{C}[G] .
$$

При этом изоморфизме каждой функции $f \in V_{\chi}$ соответствует сечение $\gamma_{f} \in$ $\Gamma(L(-\chi))$, задаваемое формулой $\gamma_{f}(g H)=[g, f(g)]$, где $[g, f(g)]$ - класс пары $(g, f(g))$ в $L(-\chi)$. Легко видеть, что пространство $V_{0} \simeq \Gamma(L(0))$, отвечающее 
характеру $\chi=0$, есть не что иное, как пространство $\mathbb{C}[G]^{H}=\mathbb{C}[G / H]$ регулярных функций на $G / H$. Отметим, что $\bigoplus_{\chi \in \mathfrak{X}(H)} V_{\chi}=\mathbb{C}[G]^{H_{0}}$, где подгруппа $H_{0} \subset H-$ пересечение ядер всех характеров группы $H$.

Пусть $\lambda \in \Lambda_{+}(G), \chi \in \mathfrak{X}(H)$. Функцию $f \in \mathbb{C}[G]$ назовем $(B \times H)$-полуинвариантной веса $(\lambda, \chi)$, если $f$ полуинвариантна веса $\lambda$ относительно действия группы $B$ слева и полуинвариантна веса $\chi$ относительно действия группы $H$ справа, т. е. $f\left(b^{-1} g h\right)=\lambda(b) \chi(h) f(g)$ при всех $b \in B, g \in G, h \in H$. Всякая ненулевая $(B \times H)$-полуинвариантная функция веса $(\lambda, \chi)$ является старшим вектором некоторого неприводимого $G$-подмодуля в $V_{\chi}$ со старшим весом $\lambda$, и наоборот. Подпространство в $\mathbb{C}[G]$, состоящее из всех $(B \times H)$-полуинвариантных функций веса $(\lambda, \chi)$, обозначим через $A(\lambda, \chi)$. Легко видеть, что кратность вхождения неприводимого $G$-модуля со старшим весом $\lambda$ в $G$-модуль $V_{\chi}$ равна $\operatorname{dim} A(\lambda, \chi)$.

Определение 1. Расширенной полугруппой старших весов $\widehat{\Lambda}_{+}(G / H)$ однородного пространства $G / H$ называется множество пар $(\lambda, \chi)\left(\lambda \in \Lambda_{+}(G)\right.$, $\chi \in \mathfrak{X}(H))$, для которых $\operatorname{dim} A(\lambda, \chi) \geqslant 1$.

Из включения $A\left(\lambda_{1}, \chi_{1}\right) A\left(\lambda_{2}, \chi_{2}\right) \subset A\left(\lambda_{1}+\lambda_{2}, \chi_{1}+\chi_{2}\right)$ вытекает, что множество $\widehat{\Lambda}_{+}(G / H)$ действительно является полугруппой. Отметим, что эта полугруппа всегда содержит элемент $(0,0)$, поскольку в пространстве $V_{0}=\mathbb{C}[G / H]$ регулярных функций на $G / H$ всегда содержатся константы. Таким образом, полугруппа $\widehat{\Lambda}_{+}(G / H)$ является моноидом.

Легко видеть, что $\Lambda_{+}(G / H) \simeq\left\{(\lambda, \chi) \in \widehat{\Lambda}_{+}(G / H) \mid \chi=0\right\} \subset \widehat{\Lambda}_{+}(G / H)$. В частности, если $\mathfrak{X}(H)=0$, то $\Lambda_{+}(G / H) \simeq \widehat{\Lambda}_{+}(G / H)$.

Напомним, что имеется следующий изоморфизм $(G \times G)$-модулей:

$$
\mathbb{C}[G] \simeq \bigoplus_{\lambda \in \Lambda_{+}(G)} V\left(\lambda^{*}\right) \otimes V(\lambda),
$$

где в левой части $G \times G$ действует слева и справа, а в каждом слагаемом правой части левый (соответственно правый) множитель группы $G \times G$ действует на левом (соответственно правом) тензорном сомножителе. Отметим, что при фиксированном $\lambda \in \Lambda_{+}(G)$ вложение $(G \times G)$-модуля $V\left(\lambda^{*}\right) \otimes V(\lambda)$ в $\mathbb{C}[G]$ осуществляется следующим образом: элементу $u \otimes v \in V\left(\lambda^{*}\right) \otimes V(\lambda)$ соответствует функция, значение которой в точке $g \in G$ равно $\langle u, g v\rangle$, где $\langle\cdot, \cdot\rangle$ - естественное спаривание между $V\left(\lambda^{*}\right)$ и $V(\lambda)$. При изоморфизме $(1)$ подпространству $A\left(\lambda^{*}, \chi\right) \subset \mathbb{C}[G]$ соответствует подпространство $v_{\lambda^{*}} \otimes V(\lambda)_{\chi}^{(H)} \subset V\left(\lambda^{*}\right) \otimes V(\lambda)$, где $V(\lambda)_{\chi}^{(H)} \subset V(\lambda)$ - подпространство $H$-полуинвариантных векторов веса $\chi$. Отсюда следует, что $\operatorname{dim} A\left(\lambda^{*}, \chi\right)=\operatorname{dim} V(\lambda)_{\chi}^{(H)}$ и $\left(\lambda^{*}, \chi\right) \in \widehat{\Lambda}_{+}(G / H)$ тогда и только тогда, когда $V(\lambda)_{\chi}^{(H)} \neq 0$.

1.3. Теперь пусть $H \subset G$ - сферическая подгруппа. В этом случае из теоремы 1 следует, что условие $\left(\lambda^{*}, \chi\right) \in \widehat{\Lambda}_{+}(G / H)$ равносильно любому из условий $\operatorname{dim} A\left(\lambda^{*}, \chi\right)=1$ или $\operatorname{dim} V(\lambda)_{\chi}^{(H)}=1$.

Важную априорную информацию о полугруппе $\widehat{\Lambda}_{+}(G / H)$ сообщает

Теорема 2. Если группа $G$ односвязна, то для всякой сферической подгрупnъь $H \subset G$ nолугруппа $\widehat{\Lambda}_{+}(G / H)$ свободна. Точнее, полугруппа $\widehat{\Lambda}_{+}(G / H)$ изоморфна полугруппе $\mathcal{D}(G / H)$ эффективных $B$-инвариантных дивизоров в $G / H$, 
свободно порожденной конечным количеством $B$-инвариантных простых дивизоров в $\mathrm{G} / \mathrm{H}$.

В случае связной сферической подгруппы $H \subset G$ тот факт, что полугруппа $\widehat{\Lambda}_{+}(G / H)$ свободна, может быть доказан при помощи рассуждения, принадлежащего Д. И. Панюшеву, хотя сам он в своей работе [6] применил его в более узкой ситуации - для доказательства того, что полугруппа $\Lambda_{+}(G / H)$ свободна при $\mathfrak{X}(H)=0$ (см. [6, предложение 2]). Адаптацию этого рассуждения к рассматриваемой ситуации см. в [2, теорема 1]. Приводимое ниже доказательство теоремы 2 в общем случае сообщено авторам Д. А. Тимашёвым.

Доказательство теоремы 2. Рассмотрим отображение $\rho: \widehat{\Lambda}_{+}(G / H) \rightarrow$ $\mathcal{D}(G / H)$, заданное следующим образом. Элементу $(\lambda, \chi) \in \widehat{\Lambda}_{+}(G / H)$ сопоставим дивизор нулей (произвольного) ненулевого сечения из одномерного подпространства $A(\lambda, \chi) \subset V_{\chi} \simeq \Gamma(L(-\chi))$. Легко видеть, что этот дивизор $B$-инвариантен и что отображение $\rho$ является гомоморфизмом полугрупп. Обратно, пусть $D \in \mathcal{D}(G / H)$ - произвольный дивизор. Как и всякий дивизор Вейля на $G / H$, дивизор $D$ является локально главным и потому определяет линейное расслоение $L$ над $G / H$ вместе с сечением $s$ (определенным однозначно с точностью до пропорциональности) таким образом, что $D$ является дивизором сечения $s$. Поскольку дивизор $D$ эффективен, сечение $s$ регулярно. Далее, в силу односвязности группы $G$ ее действие на $G / H$ «поднимается» до действия на $L$, т. е. расслоение $L$ является однородным (см. $[8$, предложение 1 и теорема 4]). Из $B$-инвариантности дивизора $D$ получаем, что сечение $s$ является $B$-полуинвариантным. Отсюда следует, что $\rho$ - биекция и, следовательно, изоморфизм.

Замечание 2. Поскольку для односвязной группы $G$ алгебра $\mathbb{C}[G]$ факториальна (см. [8, следствие из предложения 1]), в условиях теоремы 2 полугруппа $\widehat{\Lambda}_{+}(G / H)$ изоморфна также полугруппе эффективных $(B \times H)$-инвариантных дивизоров в $G$. При этом изоморфизме элементу $(\lambda, \chi) \in \widehat{\Lambda}_{+}(G / H)$ соответствует дивизор нулей (любой) ненулевой $(B \times H)$-полуинвариантной функции из $A(\lambda, \chi)$. В случае связной подгруппы $H$ полугруппа эффективных $(B \times H)$-инвариантных дивизоров в $G$ свободно порождается конечным множеством простых $(B \times H)$-инвариантных дивизоров. При этом элемент $(\lambda, \chi) \in \widehat{\Lambda}_{+}(G / H)$ неразложим тогда и только тогда, когда соответствующая ему ненулевая $(B \times H)$-полуинвариантная функция из $A(\lambda, \chi)$ неприводима в $\mathbb{C}[G]$.

\section{4. Некоторые обозначения и соглашения.}

$e$ - единичный элемент произвольной группы;

$|X|$ - мощность конечного множества $X$;

$V^{*}$ - пространство линейных функций на векторном пространстве $V$;

$\operatorname{diag}\left(a_{1}, \ldots, a_{n}\right)$ - диагональная матрица порядка $n$ с элементами $a_{1}, \ldots, a_{n}$ на диагонали.

Для группы $L$ запись $L=L_{1}<L_{2}$ означает, что $L$ разлагается в полупрямое произведение своих подгрупп $L_{1}, L_{2}$, т. е. $L=L_{1} L_{2}, L_{1} \cap L_{2}=\{e\}$ и подгруппа $L_{2}$ нормальна в $L$.

Отождествим решетку $\mathfrak{X}(T)$ с подрешеткой в $\mathfrak{t}^{*}$, сопоставив каждому характеру $\mu \in \mathfrak{X}(T)$ его дифференциал $d \mu \in \mathfrak{t}^{*}$.

Зафиксируем в $\mathfrak{X}(T)$ (и, тем самым, в $\mathfrak{t}^{*}$ ) систему корней $\Delta$ относительно $T$ и систему положительных корней $\Delta_{+} \subset \Delta$ по отношению к $B$. Пусть $\Pi=$ 
$\left\{\alpha_{1}, \ldots, \alpha_{n}\right\} \subset \Delta_{+}-$система простых корней, и пусть $\omega_{1}, \ldots, \omega_{n} \in \mathfrak{X}(T) \otimes_{\mathbb{Z}} \mathbb{Q}-$ фундаментальные веса, отвечающие простым корням $\alpha_{1}, \ldots, \alpha_{n}$ соответственно. В случае односвязной группы $G$ имеем $\omega_{1}, \ldots, \omega_{n} \in \mathfrak{X}(T)$.

Для всякого корня $\alpha \in \sum_{\gamma \in \Pi} k_{\gamma} \gamma \in \Delta_{+}$положим $\operatorname{Supp} \alpha=\left\{\gamma \mid k_{\gamma}>0\right\}$.

Пусть $W=N_{G}(T) / T$ - группа Вейля. Зафиксируем в пространстве $\mathfrak{X}(T) \otimes_{\mathbb{Z}} \mathbb{Q}$ скалярное произведение $(\cdot, \cdot)$, инвариантное относительно $W$. При $\lambda, \mu \in \mathfrak{X}(T)(\mu \neq 0)$ положим $\langle\lambda \mid \mu\rangle=2(\lambda, \mu) /(\mu, \mu)$.

Пусть $\Delta^{\vee} \subset \mathfrak{t}-$ система корней, двойственная к $\Delta$. Для каждого корня $\alpha \in \Delta$ обозначим через $h_{\alpha}$ соответствующий ему элемент из $\Delta^{\vee}$.

$\mathrm{B}$ каждом корневом подпространстве $\mathfrak{g}_{\alpha} \subset \mathfrak{g}$ выберем базисный вектор $e_{\alpha}$ так, чтобы для всякого $\alpha \in \Delta$ выполнялось условие $\left[e_{\alpha}, e_{-\alpha}\right]=h_{\alpha}$.

Благодарности. Авторы глубоко признательны Э. Б. Винбергу и Д. А. Тимашёву за прочтение предыдущей версии работы и ценные замечания.

\section{§2. Формулировка основного результата}

2.1. Для того чтобы сформулировать основную теорему, нам потребуются некоторые сведения из структурной теории связных разрешимых сферических подгрупп в полупростых алгебраических группах, см. [7].

Пусть $H \subset B-$ связная разрешимая подгруппа и $N \subset U-$ ее унипотентный радикал. Будем говорить, что подгруппа $H$ стандартно вложена в $B$ (по отношению к $T$ ), если подгруппа $S=H \cap T \subset T$ является максимальным тором в $H$. В этой ситуации, очевидно, имеем $H=S<N$. Всякую связную разрешимую подгруппу в $G$ при помощи сопряжения подходящим элементом из $G$ можно перевести в подгруппу, которая стандартно вложена в $B$.

Пусть фиксирована стандартно вложенная в $B$ связная разрешимая подгруппа $H \subset G$. Как и выше, положим $S=H \cap T$ и $N=H \cap U$, так что $H=S \curlywedge N$. Группы $\mathfrak{X}(H)$ и $\mathfrak{X}(S)$ будем отождествлять посредством ограничения характеров с $H$ на $S$. Обозначим через $\tau: \mathfrak{X}(T) \rightarrow \mathfrak{X}(S)$ отображение ограничения характеров тора $T$ на $S$. Пусть $\Phi=\tau\left(\Delta_{+}\right) \subset \mathfrak{X}(S)-$ система весов действия тора $S$ на и посредством присоединенного представления группы $G$. Имеем $\mathfrak{u}=\bigoplus_{\varphi \in \Phi} \mathfrak{u}_{\varphi}$, где $\mathfrak{u}_{\varphi} \subset \mathfrak{u}-$ весовое подпространство веса $\varphi$ относительно $S$. Пусть $\mathfrak{n}=\bigoplus_{\varphi \in \Phi} \mathfrak{n}_{\varphi}-$ разложение алгебры $\mathfrak{n}$ в прямую сумму весовых подпространств относительно тора $S$; при этом для всякого $\varphi \in \Phi$ имеем $\mathfrak{n}_{\varphi} \subset \mathfrak{u}_{\varphi}$. Для каждого $\varphi \in \Phi$ обозначим через $c_{\varphi}$ коразмерность пространства $\mathfrak{n}_{\varphi}$ в пространстве $\mathfrak{u}_{\varphi}$.

Во введенных обозначениях имеет место следующий критерий сферичности связной разрешимой подгруппы в $G$.

Теорема 3 [7, теорема 1]. Пусть $H \subset G-$ связная разрешимая подгруппа, стандартно вложенная в B. Тогда следующие условия эквивалентны:

(1) подгруппа $H$ является сферической в $G$;

(2) для всякого $\varphi \in \Phi$ выполнено неравенство $c_{\varphi} \leqslant 1$, и все веса с условием $c_{\varphi}=1$ линейно независимы в $\mathfrak{X}(S)$.

Далее мы предполагаем, что $H \subset G$ - связная разрешимая сферическая подгруппа, стандартно вложенная в $B$, и сохраняем все введенные выше обозначения. Положим $\Psi=\left\{\alpha \in \Delta_{+} \mid \mathfrak{g}_{\alpha} \not \subset \mathfrak{n}\right\} \subset \Delta_{+}$.

Определение 2. Корни из множества $\Psi$ называются активными. 
Обозначим все веса $\varphi \in \Phi$, для которых $c_{\varphi}=1$, через $\varphi_{1}, \ldots, \varphi_{m}$. При $i=$ $1, \ldots, m$ положим $\Psi_{i}=\left\{\alpha \in \Psi \mid \tau(\alpha)=\varphi_{i}\right\}$ и $\mathfrak{u}_{i}=\bigoplus_{\alpha \in \Psi_{i}} \mathfrak{g}_{\alpha}$. Очевидно, что $\Psi=\Psi_{1} \cup \cdots \cup \Psi_{m}$ и $\mathfrak{u}_{i} \subset \mathfrak{u}_{\varphi_{i}}$.

Множество активных корней обладает следующим свойством (см. [7, лемма 4]): если $\alpha$ - активный корень и $\alpha=\beta+\gamma$ для некоторых корней $\beta, \gamma \in \Delta_{+}$, то ровно один из корней $\beta, \gamma$ является активным.

Определение 3. Будем говорить, что активный корень $\beta$ подчинен активному корню $\alpha$, если существует такой корень $\gamma \in \Delta_{+}$, что $\alpha=\beta+\gamma$.

Предложение 1 [7, предложение 3]. Пусть $\alpha$ - активный коренъ. Тогда существует единственный простой корень $\pi(\alpha) \in \operatorname{Supp} \alpha$, обладающий следующим свойством: если $\alpha=\alpha_{1}+\alpha_{2}$ для некоторых корней $\alpha_{1}, \alpha_{2} \in \Delta_{+}$, то из двух корней $\alpha_{1}, \alpha_{2}$ активным является тот и только тот, носитель которого не содержит $\pi(\alpha)$.

Таким образом, имеется отображение $\pi: \Psi \rightarrow \Pi$.

При каждом $j=1, \ldots, m$ рассмотрим множество $\pi\left(\Psi_{j}\right)$. Обозначим через $\alpha_{j_{1}}, \ldots, \alpha_{j_{r}}$, где $r=r(j)=\left|\pi\left(\Psi_{j}\right)\right|$, все корни, входящие в $\pi\left(\Psi_{j}\right)$. Положим $\lambda_{j}=\omega_{j_{1}}+\cdots+\omega_{j_{r}}$.

Теперь мы можем сформулировать основной результат настоящей работы.

Теорема 4. Если группа $G$ односвязна, то полугруппа $\widehat{\Lambda}_{+}(G / H)$ свободно порождается элементами $\left(\omega_{i}^{*}, \tau\left(\omega_{i}\right)\right), i=1, \ldots, n, u$ элементами $\left(\lambda_{j}^{*}, \tau\left(\lambda_{j}\right)-\right.$ $\left.\varphi_{j}\right), j=1, \ldots, m$.

Эта теорема будет доказана в 44 .

2.2. Приведем два примера применения теоремы 4. В обоих случаях сферичность подгруппы $H$ вытекает из теоремы 3 .

Пример 1 [5]. Пусть $G$ односвязна и $H=T U^{\prime}$. Тогда $S=T, N=U^{\prime}$, $\mathfrak{n}=\bigoplus_{\alpha \in \Delta_{+} \backslash \Pi} \mathfrak{g}_{\alpha}, \tau=\mathrm{id}, m=n, \Psi=\Pi, \pi=\mathrm{id}$. Полугруппа $\widehat{\Lambda}_{+}(G / H)$ свободно порождается элементами $\left(\omega_{i}^{*}, \omega_{i}\right),\left(\omega_{i}^{*}, \omega_{i}-\alpha_{i}\right)$, где $i=1, \ldots, n$.

Пример 2. Пусть $G=\mathrm{SL}_{4}$ и группы $B, U$ и $T$ состоят из всех соответственно верхнетреугольных, верхних унитреугольных и диагональных матриц, содержащихся в $G$. При $t=\operatorname{diag}\left(t_{1}, t_{2}, t_{3}, t_{4}\right) \in T$ и $k=1,2,3$ положим $\alpha_{k}(t)=t_{k} t_{k+1}^{-1}$. Рассмотрим подгруппу $H=S<N \subset G$, где $S=\left\{\operatorname{diag}\left(s_{1}, s_{2}, s_{2}^{-1}, s_{1}^{-1}\right) \mid s_{1}, s_{2} \in\right.$ $\left.\mathbb{C}^{\times}\right\}$, а $\mathfrak{n} \subset \mathfrak{u}$ - множество всех матриц вида

$$
\left(\begin{array}{cccc}
0 & a & b & c \\
0 & 0 & d & b \\
0 & 0 & 0 & -a \\
0 & 0 & 0 & 0
\end{array}\right)
$$

с произвольными числами $a, b, c, d \in \mathbb{C}$. Отметим, что подгруппа $H$ является борелевской подгруппой в группе $\mathrm{Sp}_{4} \subset G$, сохраняющей кососимметричную билинейную форму с матрицей

$$
\left(\begin{array}{cccc}
0 & 0 & 0 & 1 \\
0 & 0 & 1 & 0 \\
0 & -1 & 0 & 0 \\
-1 & 0 & 0 & 0
\end{array}\right)
$$


Имеем $n=3, m=2, \varphi_{1}=\tau\left(\alpha_{1}\right)=\tau\left(\alpha_{3}\right), \varphi_{2}=\tau\left(\alpha_{1}+\alpha_{2}\right)=\tau\left(\alpha_{2}+\alpha_{3}\right)$, $\Psi_{1}=\left\{\alpha_{1}, \alpha_{3}\right\}, \Psi_{2}=\left\{\alpha_{1}+\alpha_{2}, \alpha_{2}+\alpha_{3}\right\}, \pi\left(\alpha_{1}\right)=\alpha_{1}, \pi\left(\alpha_{3}\right)=\alpha_{3}, \pi\left(\alpha_{1}+\alpha_{2}\right)=$ $\pi\left(\alpha_{2}+\alpha_{3}\right)=\alpha_{2}$. При $s=\operatorname{diag}\left(s_{1}, s_{2}, s_{2}^{-1}, s_{1}^{-1}\right) \in S$ положим $\chi_{1}(s)=s_{1}$, $\chi_{2}(s)=s_{1} s_{2}$. Полугруппа $\widehat{\Lambda}_{+}(G / H)$ свободно порождается пятью элементами $\left(\omega_{3}, \tau\left(\omega_{1}\right)\right)=\left(\omega_{3}, \chi_{1}\right),\left(\omega_{2}, \tau\left(\omega_{2}\right)\right)=\left(\omega_{2}, \chi_{2}\right),\left(\omega_{1}, \tau\left(\omega_{3}\right)\right)=\left(\omega_{1}, \chi_{1}\right),\left(\omega_{1}+\omega_{3}\right.$, $\left.\tau\left(\omega_{1}+\omega_{3}\right)-\varphi_{1}\right)=\left(\omega_{1}+\omega_{3}, \chi_{2}\right),\left(\omega_{2}, \tau\left(\omega_{2}\right)-\varphi_{2}\right)=\left(\omega_{2}, 0\right)$.

\section{§3. Вспомогательные результаты}

Настоящий параграф содержит все результаты, которые нам понадобятся для доказательства теоремы 4.

3.1. В этом разделе мы опишем общий метод вычисления ранга полугруппы $\widehat{\Lambda}_{+}(G / H)$ в случае произвольной связной подгруппы $H \subset G$. Этим методом мы воспользуемся в разд. 4.1 для вычисления ранга полугруппы $\widehat{\Lambda}_{+}(G / H)$ в условиях теоремы 4.

Сначала напомним одно понятие.

Пусть $L-$ редуктивная группа и $B_{L}$ - ее борелевская подгруппа. Рангом действия $L: X$ группы $L$ на неприводимом алгебраическом многообразии $X$ называется ранг решетки $\Lambda(X) \subset \mathfrak{X}\left(B_{L}\right)$, где $\Lambda(X)$ состоит из тех весов $\mu \in \mathfrak{X}\left(B_{L}\right)$, для которых в поле $\mathbb{C}(X)$ рациональных функций на $X$ содержится ненулевая $B_{L}$-полуинвариантная функция веса $\mu$. Ранг действия $L: X$ обозначим через $r_{L}(X)$. Рангом однородного пространства $L / K$ будем называть ранг естественного действия $L: L / K$ левыми сдвигами.

Пусть $H-$ произвольная связная подгруппа в $G$. Рассмотрим однородное пространство $G / H_{0}$ (определение подгруппы $H_{0}$ см. в разд. 1.2). Оно квазиаффинно, поскольку подгруппа $H_{0}$ не имеет нетривиальных характеров. На $G / H_{0}$ имеется транзитивное действие группы $\widehat{G}=G \times H / H_{0}$, где $G$ действует слева, а $H / H_{0}-$ справа. Стабилизатором точки $e H_{0}$ при этом действии является подгруппа $\widehat{H}=\left\{\left(h, h H_{0}\right) \mid h \in H\right\} \subset \widehat{G}$, изоморфная группе $H$. Таким образом, имеется изоморфизм многообразий $G / H_{0} \simeq \widehat{G} / \widehat{H}$. Алгебры $\mathbb{C}\left[G / H_{0}\right]$ и $\mathbb{C}[\widehat{G} / \widehat{H}]$ изоморфны как $\widehat{G}$-модули, поэтому имеется изоморфизм полугрупп $\widehat{\Lambda}_{+}(G / H) \simeq \Lambda_{+}(\widehat{G} / \widehat{H})$. Поскольку многообразие $\widehat{G} / \widehat{H}$ квазиаффинно, решетка $\Lambda(\widehat{G} / \widehat{H})$ порождается полугруппой $\Lambda_{+}(\widehat{G} / \widehat{H})$ (см., например, $[9$, предложение 5.14]); поэтому $\operatorname{rk} \Lambda_{+}(\widehat{G} / \widehat{H})=\operatorname{rk} \Lambda(\widehat{G} / \widehat{H})$. Отсюда $\operatorname{rk} \widehat{\Lambda}_{+}(G / H)=r_{\widehat{G}}(\widehat{G} / \widehat{H})$. Ранг однородного пространства $\widehat{G} / \widehat{H}$ можно вычислить, воспользовавшись следующим общим результатом Д. И. Панюшева.

Предложение 2 [10, теорема 1.2(ii)]. Пусть $L$ - связная редуктивная групnа, $P \subset L-$ ее параболическал подгруппа с разложением Леви $P=P_{r}<P_{u}$, где $P_{r}$ и $P_{u}$ - соответственно максимальная редуктивная подгруппа и унипотентный радикал в $P$. Пусть $K \subset L-$ связная подгруппа с разложением Леви $K=K_{r} \curlywedge K_{u}$, где $K_{r}$ и $K_{u}$ - соответственно максимальная редуктивная подгруппа и унипотентный радикал в $K$, причем $K_{r} \subset P_{r} u K_{u} \subset P_{u}$. Пусть $F \subset K_{r}-$ стабилизатор общего положения для действия $K_{r}: \mathfrak{p}_{r} / \mathfrak{k}_{r}$ (подгруппа $F$ редуктивна). Тогда $r_{L}(L / K)=r_{P_{r}}\left(P_{r} / K_{r}\right)+r_{F}\left(P_{u} / K_{u}\right)$.

3.2. В этом разделе формулируются все необходимые результаты из теории активных корней. 
Предложение 3. Пусть $1 \leqslant i, j \leqslant m$ и различные корни $\alpha \in \Psi_{i}, \beta \in \Psi_{j}$ таковы, что $\gamma=\beta-\alpha \in \Delta_{+}$. Тогда

(a) [7, предложение 1] $\Psi_{i}+\gamma \subset \Psi_{j}$;

(b) $\left[7\right.$, предложение $10($ в) $]$ если $\left|\Psi_{i}\right| \geqslant 2$, то $\gamma$ - единственный положительный корень со свойством $\Psi_{i}+\gamma \subset \Psi_{j}$.

Следствие 1. Пусть $1 \leqslant i \leqslant m$ и корни $\alpha, \beta \in \Psi_{i}$ различны. Тогда $\alpha-\beta \notin \Delta$.

Доказательство. Предположим, что $\gamma=\alpha-\beta \in \Delta$. Можно считать, что $\gamma \in \Delta_{+}$. Тогда в силу предложения $3\left(\right.$ а) имеем $\Psi_{i}+\gamma \subset \Psi_{i}$, что невозможно.

При каждом $i=1, \ldots, m$ подпространство $\mathfrak{n} \cap \mathfrak{u}_{i}$ задается в пространстве $\mathfrak{u}_{i}$ обращением в нуль некоторой (определенной с точностью до пропорциональности) линейной функции, которую обозначим через $\xi_{i}$. Если $\alpha \in \Psi_{i}$ для некоторого $i \in\{1, \ldots, m\}$, то ограничение функции $\xi_{i}$ на $\mathfrak{g}_{\alpha}$ не равно нулю.

Предложение 4 [7, предложение 2]. Если $\Psi_{i}+\gamma \subset \Psi_{j}$ для некоторых $i, j \in$ $\{1, \ldots, m\}$ и $\gamma \in \Delta_{+}$, то существует такое число $c \neq 0$, ито $\xi_{i}(x)=c \xi_{j}\left(\left[x, e_{\gamma}\right]\right)$ при всех $x \in \mathfrak{u}_{i}$.

Предложение 5 [7, следствие 11]. Пусть активные корни $\alpha, \beta$ таковъ, что $\tau(\alpha)=\tau(\beta)$. Тогда либо $\pi(\alpha)=\pi(\beta)$, либо ни один из корней $\pi(\alpha), \pi(\beta)$ не содержится в $\operatorname{Supp} \alpha \cap \operatorname{Supp} \beta$.

\section{§4. Доказательство основной теоремы}

C учетом теоремы 2 доказательство теоремы 4 включает в себя три этапа. Сначала в разд. 4.1 мы вычислим ранг полугруппы $\widehat{\Lambda}_{+}(G / H)$. Затем в разд. 4.2 мы покажем, что все элементы, указанные в формулировке теоремы 4, действительно содержатся в этой полугруппе. Наконец, в разд. 4.3 мы докажем, что указанные элементы неразложимы в $\widehat{\Lambda}_{+}(G / H)$.

В этом параграфе мы сохраняем обозначения, введенные в разд. 2.1.

4.1. В этом разделе мы докажем следующее

Предложение 6. В условиях теоремъ 4 ранг полугруппы $\widehat{\Lambda}_{+}(G / H)$ равен $n+m$.

Ниже приводятся два различных доказательства этого предложения. В первом из них применяется общий метод вычисления ранга расширенной полугруппы старших весов, описанный в разд. 3.1. Второе доказательство является прямым и использует геометрические соображения.

Доказательство 1. При естественном эпиморфизме $H \rightarrow H / H_{0}$ подгруппа $S \subset H$ изоморфно отображается на $H / H_{0}$. Ввиду этого далее мы будем отождествлять группы $S$ и $H / H_{0}$. В разд. 3.1 было показано, что $\operatorname{rk} \widehat{\Lambda}_{+}(G / H)=$ $r_{\widehat{G}}(\widehat{G} / \widehat{H})$. Для вычисления $r_{\widehat{G}}(\widehat{G} / \widehat{H})$ применим предложение 2 при $L=\widehat{G}=$ $G \times S$ и $K=\widehat{H}$. Положим $P=B \times S$. Тогда $P_{r}=T \times S, P_{u}=U ; K_{r}=\widehat{S}$, $K_{u}=N$, где подгруппа $\widehat{S} \subset \widehat{H}$ изоморфна $S$ и вложена в подгруппу $S \times S \subset$ $H \times S$ диагонально. Подгруппа $\widehat{S}$ тривиально действует в пространстве $\mathfrak{p}_{r} / \mathfrak{k}_{r} \simeq$ $(\mathfrak{t} \oplus \mathfrak{s}) / \widehat{\mathfrak{s}}$, поэтому $F=\widehat{S}$. Следовательно, $r_{\widehat{G}}(\widehat{G} / \widehat{H})=r_{T \times S}((T \times S) / \widehat{S})+$ $r_{\widehat{S}}(U / N)$. Легко видеть, что в последней сумме первое слагаемое равно $\operatorname{rk} T=n$. Найдем значение второго слагаемого этой суммы. Ясно, что оно совпадает с $r_{S}(U / N)$. В силу [11, лемма 1.4] имеется $S$-эквивариантный изоморфизм $U / N \simeq$ 
$\mathfrak{u} / \mathfrak{n}$. По теореме 3 пространство $\mathfrak{u} / \mathfrak{n}$ как $S$-модуль изоморфно прямой сумме $\mathbb{C}_{\varphi_{1}} \oplus \cdots \oplus \mathbb{C}_{\varphi_{m}}$, где $\mathbb{C}_{\varphi_{i}} \simeq \mathbb{C}$ - подпространство веса $\varphi_{i}$ относительно $S$, причем веса $\varphi_{1}, \ldots, \varphi_{m}$ линейно независимы. Отсюда вытекает, что $r_{S}(U / N)=m$.

Доказательство 2. Поскольку подгруппа $H$ связна, с учетом замечания 2 получаем, что ранг полугруппы $\widehat{\Lambda}_{+}(G / H)$ равен количеству простых $(B \times H)$-инвариантных дивизоров в $G$. Рассмотрим разложение Брюа группы $G$ :

$$
G=\bigsqcup_{\sigma \in W} B \sigma B
$$

где объединение является дизъюнктным. Среди подмножеств $B \sigma B \subset G$, каждое из которых $(B \times B)$-инвариантно, имеется открытое подмножество $G_{0}=$ $B \sigma_{0} B \subset G$ (называемое открытой клеткой), где $\sigma_{0}$ - элемент наибольшей длины в группе $W$. Хорошо известно, что дополнение в $G$ множества $G_{0}$ является объединением ровно $n$ простых дивизоров, которые являются $(B \times B)$-инвариантными, а значит, и $(B \times H)$-инвариантными. Поэтому для завершения доказательства остается доказать, что в $G_{0}$ имеется ровно $m$ простых $(B \times H)$-инвариантных дивизоров. С учетом изоморфизма $G_{0} \simeq B \sigma_{0} \times U$ мы получаем, что $(B \times H)$-инвариантные дивизоры в $G_{0}$ находятся во взаимно однозначном соответствии с $H$-инвариантными дивизорами в $U$, где действие $H$ на $U$ описывается формулой $(s v, u) \mapsto s u v^{-1} s^{-1}(s \in S, v \in N, u \in U)$. В свою очередь, $H$-инвариантные дивизоры в $U$ находятся во взаимно однозначном соответствии с $S$-инвариантными дивизорами в $U / N$ или, с учетом $S$-эквивариантного изоморфизма $U / N \simeq \mathfrak{u} / \mathfrak{n}$ (см. [11, лемма 1.4]), с $S$-инвариантными дивизорами в $\mathfrak{u} / \mathfrak{n}$. Принимая во внимание изоморфизм $S$-модулей $\mathfrak{u} / \mathfrak{n} \simeq \mathbb{C}_{\varphi_{1}} \oplus \cdots \oplus \mathbb{C}_{\varphi_{m}}$ (см. теорему 3 ), мы получаем, что простые $S$-инвариантные дивизоры в $\mathfrak{u} / \mathfrak{n}-$ это в точности дивизоры нулей $S$-полуинвариантных линейных функций на $\mathfrak{u} / \mathfrak{n}$, которых с точностью до пропорциональности ровно $m$.

4.2. В этом разделе мы покажем, что элементы, перечисленные в формулировке теоремы 4 , содержатся в полугруппе $\widehat{\Lambda}_{+}(G / H)$.

Как отмечалось ранее (см. разд. 1.2$)$, условие $\left(\lambda^{*}, \chi\right) \in \widehat{\Lambda}_{+}(G / H)$ эквивалентно тому, что $V(\lambda)_{\chi}^{(H)} \neq 0$. Для элементов $\left(\omega_{i}^{*}, \tau\left(\omega_{i}\right)\right)$ при $i=1, \ldots, n$ указать ненулевой вектор в $V\left(\omega_{i}\right)_{\tau\left(\omega_{i}\right)}^{(H)}$ совсем просто: таковым будет старший вектор $v_{\omega_{i}} \in V\left(\omega_{i}\right)$. Теперь остается доказать, что $\left(\lambda_{j}^{*}, \tau\left(\lambda_{j}\right)-\varphi_{j}\right) \in \Lambda_{+}(G / H)$ или, эквивалентно, $V\left(\lambda_{j}\right)_{\tau\left(\lambda_{j}\right)-\varphi_{j}}^{(H)} \neq 0$ при всех $j=1, \ldots, m$. Этому посвящена оставшаяся часть раздела.

Ниже нам потребуется следующая простая

Лемма 1. Пусть $\mu \in \Lambda_{+}(G) u \alpha, \beta \in \Delta_{+}$. Тогда

(a) $e_{\alpha}\left(e_{-\beta} v_{\mu}\right)=\left[e_{\alpha}, e_{-\beta}\right] v_{\mu}$;

(b) если $\left[e_{\alpha}, e_{-\beta}\right] v_{\mu} \neq 0$, mo $\beta-\alpha \in \Delta_{+}$.

Зафиксируем $j \in\{1, \ldots, m\}$. Обозначим через $\beta_{1}, \ldots, \beta_{p}$ все корни, входящие в $\Psi_{j}$. Линейная функция $\xi_{j} \in \mathfrak{u}_{j}^{*}$ (см. разд. 3.2 ) задается набором ненулевых чисел $a_{1}, \ldots, a_{p}$ следующим образом: элемент $x_{1} e_{\beta_{1}}+\cdots+x_{p} e_{\beta_{p}} \in \mathfrak{u}_{j}$ лежит в $\mathfrak{n}$ тогда и только тогда, когда $a_{1} x_{1}+\cdots+a_{p} x_{p}=0$.

Пусть $\pi\left(\Psi_{j}\right)=\left\{\alpha_{j_{1}}, \ldots, \alpha_{j_{r}}\right\}$ и $\lambda_{j}=\omega_{j_{1}}+\cdots+\omega_{j_{r}} \in \Lambda_{+}(G)$ (см. разд. 2.1). Отметим, что при каждом $k=1, \ldots, p$ в силу условия $\pi\left(\beta_{k}\right) \in \operatorname{Supp} \beta_{k}$ имеем 
$\lambda_{j}\left(h_{\beta_{k}}\right)=\left\langle\lambda_{j} \mid \beta_{k}\right\rangle>0$ и, значит, $e_{-\beta_{k}} v_{\lambda_{j}} \neq 0$. Положим

$$
f_{j}=\frac{a_{1}}{\lambda_{j}\left(h_{\beta_{1}}\right)} e_{-\beta_{1}}+\cdots+\frac{a_{p}}{\lambda_{j}\left(h_{\beta_{p}}\right)} e_{-\beta_{p}} \in \mathfrak{g}
$$

и рассмотрим в неприводимом $G$-модуле $V\left(\lambda_{j}\right)$ со старшим весом $\lambda_{j}$ (ненулевой) вектор $w_{j}=f_{j} v_{\lambda_{j}} \in V\left(\lambda_{j}\right)$.

Покажем, что $w_{j} \in V\left(\lambda_{j}\right)_{\tau\left(\lambda_{j}\right)-\varphi_{j}}^{(H)}$. Легко видеть, что вектор $w_{j}$ является $S$-полуинвариантным веса $\tau\left(\lambda_{j}\right)-\varphi_{j}$. Докажем, что этот вектор является $H$-полуинвариантным. Для этого достаточно доказать следующее

Предложение 7. Вектор $w_{j}$ аннулируется алгеброй $\mathfrak{n}$.

Доказательство состоит из трех шагов.

Шаг 1. Докажем, что вектор $w_{j}$ аннулируется подпространством $\mathfrak{n} \cap \mathfrak{u}_{j} \cdot$ Для этого возьмем произвольный элемент $x=x_{1} e_{\beta_{1}}+\cdots+x_{p} e_{\beta_{p}} \in \mathfrak{n} \cap \mathfrak{u}_{j}$ и покажем, что $x w_{j}=0$. С учетом леммы $1(\mathrm{a})$ имеем

$$
x w_{j}=\left(\sum_{k=1}^{p} x_{k} e_{\beta_{k}}\right)\left(\sum_{l=1}^{p} \frac{a_{l}}{\lambda_{j}\left(h_{\beta_{l}}\right)} e_{-\beta_{l}}\right) v_{\lambda_{j}}=\sum_{k=1}^{p} \sum_{l=1}^{p} \frac{a_{l} x_{k}}{\lambda_{j}\left(h_{\beta_{l}}\right)}\left[e_{\beta_{k}}, e_{-\beta_{l}}\right] v_{\lambda_{j}} .
$$

При всех $k \neq l$ в силу следствия 1 имеем $\beta_{k}-\beta_{l} \notin \Delta$, откуда вытекает, что $\left[e_{\beta_{k}}, e_{-\beta_{l}}\right]=0$. Значит,

$$
x w_{j}=\sum_{k=1}^{p} \frac{a_{k} x_{k}}{\lambda_{j}\left(h_{\beta_{k}}\right)}\left[e_{\beta_{k}}, e_{-\beta_{k}}\right] v_{\lambda_{j}}=\sum_{k=1}^{p} \frac{a_{k} x_{k}}{\lambda_{j}\left(h_{\beta_{k}}\right)} h_{\beta_{k}} v_{\lambda_{j}}=\left(\sum_{k=1}^{p} a_{k} x_{k}\right) v_{\lambda_{j}}=0
$$

где учтено, что $h_{\beta_{k}} v_{\lambda_{j}}=\lambda_{j}\left(h_{\beta_{k}}\right) v_{\lambda_{j}}$ и $a_{1} x_{1}+\cdots+a_{p} x_{p}=0$.

Шаг 2. Докажем, что вектор $w_{j}$ аннулируется подпространством $\mathfrak{n} \cap \mathfrak{u}_{i}$ при $i \neq j$. Пусть $\Psi_{i}=\left\{\gamma_{1}, \ldots, \gamma_{q}\right\}$. Если $\left|\Psi_{i}\right|=1$, то $\mathfrak{n} \cap \mathfrak{u}_{i}=\{0\}$ и доказывать нечего. Поэтому далее считаем, что $\left|\Psi_{i}\right| \geqslant 2$. Линейная функция $\xi_{i} \in \mathfrak{u}_{i}^{*}$ задается набором ненулевых чисел $b_{1}, \ldots, b_{q}$ так, что элемент $y_{1} e_{\gamma_{1}}+\cdots+y_{q} e_{\gamma_{q}}$ лежит в $\mathfrak{n}$ тогда и только тогда, когда $b_{1} y_{1}+\cdots+b_{q} y_{q}=0$. Пусть $y=y_{1} e_{\gamma_{1}}+\cdots+y_{q} e_{\gamma_{q}} \in$ $\mathfrak{n} \cap \mathfrak{u}_{i}$. Покажем, что $y w_{j}=0$. С учетом леммы $1(\mathrm{a})$ имеем

$$
y w_{j}=\left(\sum_{k=1}^{q} y_{k} e_{\gamma_{k}}\right)\left(\sum_{l=1}^{p} \frac{a_{l}}{\lambda_{j}\left(h_{\beta_{l}}\right)} e_{-\beta_{l}}\right) v_{\lambda_{j}}=\sum_{k=1}^{q} \sum_{l=1}^{p} \frac{a_{l} y_{k}}{\lambda_{j}\left(h_{\beta_{l}}\right)}\left[e_{\gamma_{k}}, e_{-\beta_{l}}\right] v_{\lambda_{j}} .
$$

Предположим, что при некоторых $k \in\{1, \ldots, q\}$ и $l \in\{1, \ldots, p\}$ элемент $\left[e_{\gamma_{k}}, e_{-\beta_{l}}\right]$ нетривиально действует на вектор $v_{\lambda_{j}}$. Тогда по лемме $1(\mathrm{~b})$ получаем, что $\gamma_{k}-\beta_{l}=-\delta$ для некоторого корня $\delta \in \Delta_{+}$. В силу предложения $3($ а) имеем $\Psi_{i}+\delta \subset \Psi_{j}$. Так как $\left|\Psi_{i}\right| \geqslant 2$, то по предложению $3(\mathrm{~b})$ корень $\delta$ является единственным положительным корнем со свойством $\Psi_{i}+\delta \subset \Psi_{j}$. Перенумеровав корни в $\Psi_{j}$, без ограничения общности можно считать, что $\gamma_{1}+\delta=\beta_{1}, \ldots, \gamma_{q}+$ $\delta=\beta_{q}$. Тогда

$$
y w_{j}=\sum_{k=1}^{q} \frac{a_{k} y_{k}}{\lambda_{j}\left(h_{\beta_{k}}\right)}\left[e_{\gamma_{k}}, e_{-\beta_{k}}\right] v_{\lambda_{j}}=\left(\sum_{k=1}^{q} \frac{a_{k} d_{k} y_{k}}{\lambda_{j}\left(h_{\beta_{k}}\right)}\right) e_{-\delta} v_{\lambda_{j}},
$$

где при $k=1, \ldots, q$ числа $d_{k}$ таковы, что $\left[e_{\gamma_{k}}, e_{-\beta_{k}}\right]=d_{k} e_{-}$. Далее, при $k=$ $1, \ldots, q$ имеем $\left[e_{\gamma_{k}}, e_{\delta}\right]=c_{k} e_{\beta_{k}}$, где $c_{k} \neq 0$. Тогда с учетом предложения 4 можно считать, что $b_{k}=a_{k} c_{k}$ при всех $k=1, \ldots, q$. 
Для завершения доказательства нам достаточно показать, что

$$
\frac{a_{1} d_{1} y_{1}}{\lambda_{j}\left(h_{\beta_{1}}\right)}+\cdots+\frac{a_{q} d_{q} y_{q}}{\lambda_{j}\left(h_{\beta_{q}}\right)}=0 .
$$

Подставляя в эту формулу $a_{k}=b_{k} / c_{k}$ при $k=1, \ldots, q$ и учитывая условие $b_{1} y_{1}+\cdots+b_{q} y_{q}=0$, мы находим, что теперь нам достаточно доказать следующее:

$$
\frac{d_{1}}{c_{1} \lambda_{j}\left(h_{\beta_{1}}\right)}=\cdots=\frac{d_{q}}{c_{q} \lambda_{j}\left(h_{\beta_{q}}\right)} .
$$

Для этого при фиксированном $k \in\{1, \ldots, q\}$ рассмотрим три элемента $e_{\gamma_{k}}, e_{\delta}$, $e_{-\beta_{k}}$ и выпишем для них тождество Якоби:

$$
\left[\left[e_{\gamma_{k}}, e_{\delta}\right], e_{-\beta_{k}}\right]+\left[\left[e_{-\beta_{k}}, e_{\gamma_{k}}\right], e_{\delta}\right]+\left[\left[e_{\delta}, e_{-\beta_{k}}\right], e_{\gamma_{k}}\right]=0
$$

или

$$
c_{k} h_{\beta_{k}}+d_{k} h_{\delta}+A_{k} h_{\gamma_{k}}=0,
$$

где $A_{k}$ - некоторое число. Подействовав обеими частями этого равенства на вектор $v_{\lambda_{j}}$, получаем

$$
c_{k} \lambda_{j}\left(h_{\beta_{m}}\right)+d_{k} \lambda_{j}\left(h_{\delta}\right)+A_{k} \lambda_{j}\left(h_{\gamma_{k}}\right)=0 .
$$

Так как $\gamma_{k}$ - активный корень, подчиненный активному корню $\beta_{k}$, то простой корень $\pi\left(\beta_{k}\right)=\alpha_{j_{l}}$ не содержится в множестве Supp $\gamma_{k}$, откуда $\omega_{j_{l}}\left(h_{\gamma_{k}}\right)=0$. Далее, в силу условия Supp $\gamma_{m} \subset \operatorname{Supp} \beta_{m}$ и предложения 5 получаем, что $\pi\left(\Psi_{j}\right) \cap \operatorname{Supp} \gamma_{k}=\varnothing$. Отсюда $\omega_{j_{1}}\left(h_{\gamma_{k}}\right)=\cdots=\omega_{j_{r}}\left(h_{\gamma_{k}}\right)=0$ и $\lambda_{j}\left(h_{\gamma_{k}}\right)=0$. Таким образом, $c_{k} \lambda_{j}\left(h_{\beta_{k}}\right)+d_{k} \lambda_{j}\left(h_{\delta}\right)=0$. Заметим, что $\lambda_{j}\left(h_{\delta}\right)=\left\langle\lambda_{j} \mid \delta\right\rangle=\left\langle\omega_{j_{l}} \mid \delta\right\rangle>0$, поскольку $\alpha_{j_{l}} \in \operatorname{Supp} \delta$. Значит, величина

$$
\frac{d_{k}}{c_{k} \lambda_{j}\left(h_{\beta_{k}}\right)}=-\frac{1}{\lambda_{j}\left(h_{\delta}\right)}
$$

не зависит от $k$, что и оставалось доказать.

Шаг 3. Докажем, что вектор $w_{j}$ аннулируется подпространством $\mathfrak{g}_{\gamma}$ при любом $\gamma \in \Delta_{+} \backslash \Psi$. С учетом леммы 1 (а) имеем

$$
e_{\gamma} w_{j}=e_{\gamma}\left(\sum_{k=1}^{p} \frac{a_{k}}{\lambda_{j}\left(h_{\beta_{k}}\right)} e_{-\beta_{k}}\right) v_{\lambda_{j}}=\sum_{k=1}^{p} \frac{a_{k}}{\lambda_{j}\left(h_{\beta_{k}}\right)}\left[e_{\gamma}, e_{-\beta_{k}}\right] v_{\lambda_{j}} .
$$

Покажем, что в последней сумме каждое слагаемое равно нулю. Предположим противное: $\left[e_{\gamma}, e_{-\beta_{k}}\right] v_{\lambda_{j}} \neq 0$ при некотором $k \in\{1, \ldots, p\}$. Тогда по лемме $1(\mathrm{~b})$ получаем, что $\delta=\beta_{k}-\gamma \in \Delta_{+}$. Отсюда следует, что $e_{-\delta} v_{\lambda_{j}} \neq 0$ и $\left\langle\lambda_{j} \mid \delta\right\rangle>0$. $\mathrm{C}$ другой стороны, из условий $\beta_{k}=\gamma+\delta$ и $\gamma \notin \Psi$ вытекает, что $\delta-$ активный корень, подчиненный активному корню $\beta_{k}$. Это означает, что $\pi\left(\beta_{k}\right) \notin \operatorname{Supp} \delta$. Тогда по предложению 5 имеем $\pi\left(\Psi_{i}\right) \cap \operatorname{Supp} \delta=\varnothing$, откуда $\left\langle\lambda_{j} \mid \delta\right\rangle=0$. Полученное противоречие завершает шаг 3 и доказательство предложения.

Замечание 3. Если $p=1$ (т. е. $\left|\Psi_{j}\right|=1$ ), то доказательство предложения 7 значительно упрощается. А именно, в этом случае на шаге 1 нечего доказывать (так как $\mathfrak{n} \cap \mathfrak{u}_{j}=0$ ), а из шага 2 исчезает содержательная часть: при $i \neq j$ и $\left|\Psi_{i}\right| \geqslant 2$ условие $\Psi_{i}+\gamma \subset \Psi_{j}$ не может быть выполнено ни для какого корня $\gamma \in \Delta_{+}$. Отметим также, что при $p=1$ вектор $w_{j}$ пропорционален вектору $e_{-\beta_{1}} v_{\lambda_{j}}$. 
4.3. В этом разделе мы докажем, что элементы полугруппы $\widehat{\Lambda}_{+}(G / H)$, перечисленные в формулировке теоремы 4, неразложимы в этой полугруппе. В силу связности подгруппы $H$ и замечания 2 для этого достаточно доказать, что ненулевые $(B \times H)$-полуинвариантные регулярные функции на $G$, соответствующие указанным элементам, неприводимы в $\mathbb{C}[G]$.

При $i=1, \ldots, n$ обозначим через $P_{i}$ функцию из $\mathbb{C}[G]$, соответствующую при изоморфизме (1) элементу $v_{\omega_{i}^{*}} \otimes v_{\omega_{i}} \in V\left(\omega_{i}^{*}\right) \otimes V\left(\omega_{i}\right)$. Имеем $P_{i}(g)=$ $\left\langle v_{\omega_{i}^{*}}, g v_{\omega_{i}}\right\rangle$ при всех $g \in G$. Функция $P_{i}$ является $(B \times H)$-полуинвариантной веса $\left(\omega_{i}^{*}, \tau\left(\omega_{i}\right)\right)$. Аналогично, при $j=1, \ldots, m$ обозначим через $Q_{j}$ функцию из $\mathbb{C}[G]$, соответствующую при изоморфизме $(1)$ элементу $v_{\lambda_{j}^{*}} \otimes w_{j} \in V\left(\lambda_{j}^{*}\right) \otimes V\left(\lambda_{j}\right)$. Имеем $Q_{j}(g)=\left\langle v_{\lambda_{j}^{*}}, g w_{j}\right\rangle$ при всех $g \in G$. Функция $Q_{i}$ является $(B \times H)$-полуинвариантной веса $\left(\lambda_{j}^{*}, \tau\left(\lambda_{j}\right)-\varphi_{j}\right)$.

Лемма 2. Пусть $i \in\{1, \ldots, n\}$ u $v \in V\left(\omega_{i}\right) \backslash\{0\}$. Тогда функиия $f \in \mathbb{C}[G]$, соответствующая при изоморфизме (1) элементу $v_{\omega_{i}^{*}} \otimes v \in V\left(\omega_{i}^{*}\right) \otimes V\left(\omega_{i}\right)$, неприводима в $\mathbb{C}[G]$.

Доказательство. Рассмотрим алгебру $U \mathbb{C}[G]$. С учетом изоморфизма (1) имеем

$$
U \mathbb{C}[G] \simeq \bigoplus_{\lambda \in \Lambda_{+}(G)} v_{\lambda^{*}} \otimes V(\lambda),
$$

где компонента $v_{\lambda^{*}} \otimes V(\lambda)$ рассматривается как подпространство в $V\left(\lambda^{*}\right) \otimes$ $V(\lambda)$. Действие тора $T$ слева задает на алгебре ${ }^{U} \mathbb{C}[G]$ градуировку полугруппой $\Lambda_{+}(G)$, причем компоненте веса $\lambda^{*}$ соответствует подпространство $v_{\lambda^{*}} \otimes V(\lambda)$. Из условия следует, что функция $f$ содержится в компоненте веса $\omega_{i}^{*}$ алгебры $U \mathbb{C}[G]$. Поскольку компонента веса 0 этой алгебры состоит только из констант, а вес $\omega_{i}^{*}$ не допускает нетривиальных представлений в виде суммы двух элементов из $\Lambda_{+}(G)$, мы получаем, что функция $f$ неприводима в $U \mathbb{C}[G]$. Остается заметить, что для функции из $U \mathbb{C}[G]$ неприводимость в $U \mathbb{C}[G]$ эквивалентна неприводимости в $\mathbb{C}[G]$, поскольку группа $U$ связна и не имеет нетривиальных характеров (см. [12, теорема 3.17]).

Следствие 2. При всех $i=1, \ldots, n$ функиия $P_{i}$ неприводима в $\mathbb{C}[G]$.

Заметим, что при каждом $i=1, \ldots, n$ функция $P_{i}$ является не только $(B \times H)$-полуинвариантной, но и $(B \times B)$-полуинвариантной. Поэтому соответствующий ей простой $(B \times H)$-инвариантный дивизор $D_{i} \subset G$ является также $(B \times B)$-инвариантным. Как уже упоминалось в разд. 4.1 (см. доказательство 2 предложения 6), дополнение в $G$ открытой клетки Брюа $G_{0}=B \sigma_{0} B$ является объединением ровно $n$ простых дивизоров, являющихся $(B \times B)$-инвариантными. Ясно, что эти дивизоры - не что иное, как $D_{1}, \ldots, D_{n}$.

Для завершения доказательства теоремы 4 осталось доказать, что функция $Q_{j}$ неприводима в $\mathbb{C}[G]$ при $j=1, \ldots, m$.

Предложение 8. При всех $i=1, \ldots, n u j=1, \ldots, m$ функиия $Q_{j}$ не делится на функцию $P_{i}$.

Доказательство. Зафиксируем $i \in\{1, \ldots, n\}, j \in\{1, \ldots, m\}$ и покажем, что $Q_{j}$ не делится на $P_{i}$. Функция $Q_{j} / P_{i}$ является $(B \times H)$-полуинвариантной рациональной функцией на $G$ веса $\left(\lambda_{j}^{*}-\omega_{i}^{*}, \tau\left(\lambda_{j}-\omega_{i}\right)-\varphi_{j}\right)$. Если $i \notin\left\{j_{1}, \ldots, j_{r}\right\}$, 
то вес $\lambda_{j}^{*}-\omega_{i}^{*}$ не является доминантным, а значит, функция $Q_{j} / P_{i}$ не является регулярной. Поэтому далее предполагаем, что $i \in\left\{j_{1}, \ldots, j_{r}\right\}$. Более того, без ограничения общности можно считать, что $i=j_{1}$. Напомним, что $w_{j}=f_{j} v_{\lambda_{j}}$, где элемент $f_{j}$ определен в разд. 4.2. Функция, соответствующая при изоморфизме $(1)$ элементу $v_{\lambda_{j}^{*}} \otimes v_{\lambda_{j}}$, является $(B \times H)$-полуинвариантной веса $\left(\lambda_{j}^{*}, \lambda_{j}\right)$. В силу условия $\operatorname{dim} A\left(\lambda_{j}^{*}, \lambda_{j}\right) \leqslant 1$ получаем, что эта функция совпадает с функцией $c P_{i} P_{j_{2}} \cdots P_{j_{r}}$ для некоторой константы $c \neq 0$. Без ограничения общности считаем, что $c=1$. Тогда $Q_{j}=f_{j}\left(P_{i} P_{j_{2}} \cdots P_{j_{r}}\right)$, где действие алгебры Ли $\mathfrak{g}$ на $\mathbb{C}[G]$ индуцировано действием группы $G$ справа. Элемент $f_{j}$ действует на произведение $P_{i} P_{j_{2}} \cdots P_{j_{r}}$ как дифференцирование, поэтому $Q_{j}=\left(f_{j} P_{i}\right) P_{j_{2}} \cdots P_{j_{r}}+P_{i} \cdot f_{j}\left(P_{j_{2}} \cdots P_{j_{r}}\right)$. Отсюда следует, что делимость функции $Q_{j}$ на $P_{i}$ равносильна делимости на $P_{i}$ функции $f_{j} P_{i}$. При изоморфизме $(1)$ функции $f_{j} P_{i}$ соответствует вектор $v_{\omega_{i}^{*}} \otimes\left(f_{j} v_{\omega_{i}}\right) \in V\left(\omega_{i}^{*}\right) \otimes V\left(\omega_{i}\right)$. Покажем, что он отличен от нуля, т. е. $f_{j} v_{\omega_{i}} \neq 0$. Для этого достаточно найти такой корень $\beta \in \Psi_{j}$, что $e_{-\beta} v_{\omega_{i}} \neq 0$. Последнее условие равносильно неравенству $\left\langle\omega_{i} \mid \beta\right\rangle>0$, которое заведомо выполнено для любого корня $\beta \in \Psi_{j}$ с условием $\pi(\beta)=\alpha_{i}$. Итак, вектор $f_{j} v_{\omega_{i}} \in V\left(\omega_{i}\right)$ отличен от нуля. Легко видеть, что он не пропорционален вектору $v_{\omega_{i}}$. Отсюда с учетом леммы 2 вытекает, что функция $f_{j} P_{i}$ неприводима в $\mathbb{C}[G]$ и не пропорциональна функции $P_{i}$. Следовательно, функция $f_{j} P_{i}$, а значит и функция $Q_{j}$, не делится на $P_{i}$.

В силу условия $G \backslash G_{0}=D_{1} \cup \cdots \cup D_{n}$ и предложения 8 доказательство неприводимости в $\mathbb{C}[G]$ каждой из функций $Q_{j}$ сводится к следующему предложению.

Предложение 9. При каждом $j=1, \ldots, m$ ограничение функиии $Q_{j}$ на множество $G_{0}$ является неприводимой функиией в $\mathbb{C}\left[G_{0}\right]$.

Доказательство. Зафиксируем $j \in\{1, \ldots, m\}$. Тогда $\lambda_{j}=\omega_{j_{1}}+\cdots+\omega_{j_{r}}$. Ограничения функций $P_{1}, \ldots, P_{n}$ на $G_{0}$ являются обратимыми функциями, поэтому нам достаточно доказать, что функция $\widehat{Q}_{j}=Q_{j} /\left(P_{j_{1}} \cdots P_{j_{r}}\right)$ неприводима в $\mathbb{C}\left[G_{0}\right]$. Отметим, что эта функция $(B \times H)$-полуинвариантна веса $\left(0,-\varphi_{j}\right)$. Поскольку группа $B \times H$ имеет открытую орбиту в $G_{0}$, подпространство в $\mathbb{C}\left[G_{0}\right]$, состоящее из всех $(B \times H)$-полуинвариантных функций веса $\left(0,-\varphi_{j}\right)$, одномерно. Доказательство будет завершено, если мы предъявим в $\mathbb{C}\left[G_{0}\right]$ неприводимую $(B \times H)$-полуинвариантную функцию веса $\left(0,-\varphi_{j}\right)$. Группа $U \times N$ связна и не имеет нетривиальных характеров; поэтому в силу $[12$, теорема 3.17] нам достаточно предъявить в алгебре ${ }^{U} \mathbb{C}\left[G_{0}\right]^{N}$ неприводимую $(T \times S)$-полуинвариантную функцию веса $\left(0,-\varphi_{j}\right)$. Имеем ${ }^{U} \mathbb{C}\left[G_{0}\right]^{N} \simeq U \mathbb{C}[U \times$ $\left.T \sigma_{0} \times U\right]^{N} \simeq \mathbb{C}[T \times U / N] \simeq \mathbb{C}[T \times \mathfrak{u} / \mathfrak{n}]$, где последний изоморфизм обусловлен существованием $S$-эквивариантного изоморфизма $U / N \simeq \mathfrak{u} / \mathfrak{n}$ (см. [11, лемма 1.4]). Действие группы $T \times S$ на многообразии $T \times \mathfrak{u} / \mathfrak{n}$ устроено следующим образом. Пара $(t, s) \in T \times S$ переводит пару $\left(t_{0}, x\right) \in T \times \mathfrak{u} / \mathfrak{n}$ в пару $\left(t t_{0}\left(\sigma_{0} s^{-1} \sigma_{0}^{-1}\right), s x\right)$. Напомним, что по теореме 3 пространство $\mathfrak{u} / \mathfrak{n}$ как $S$-модуль изоморфно прямой сумме $\mathbb{C}_{\varphi_{1}} \oplus \cdots \oplus \mathbb{C}_{\varphi_{m}}$, где $\mathbb{C}_{\varphi_{i}} \simeq \mathbb{C}-$ подпространство веса $\varphi_{i}$ относительно $S$. Пусть $l_{j}$ есть $j$-я координатная функция на $\mathfrak{u} / \mathfrak{n}$. Тогда $l_{j}$, будучи линейной, является неприводимой $S$-полуинвариантной функцией веса $-\varphi_{j}$. Значит, функция $1 \otimes l_{j} \in \mathbb{C}[T] \otimes \mathbb{C}[\mathfrak{u} / \mathfrak{n}] \simeq \mathbb{C}[T \times \mathfrak{u} / \mathfrak{n}]$ является неприводимой $(T \times S)$-полуинвариантной функцией веса $\left(0,-\varphi_{j}\right)$. 


\section{ЛитЕРАТУРА}

[1] Э. Б. Винберг, Б. Н. Кимельфельд, Однородные области на флаговых многообразиях и сферические подгруппы полупростых групп Ли, Функц. анализ и его прил., 12:3 (1978), 12-19.

[2] Р. С. Авдеев, Расширенные полугруппы стариих весов афбинных сферических однородных пространств непростых полупростых алгебраических групп, Изв. РАН. Сер. матем., 74:6 (2010), 3-26.

[3] M. Krämer, Sphärische Untergruppen in kompakten zusammenhängenden Liegruppen, Compositio Math., 38:2 (1979), 129-153.

[4] F. Knop, Weylgruppe und Momentabbildung, Invent. Math., 99:1 (1990), 1-23.

[5] Н. Е. Горфинкель, Гармонический анализ на некотором классе сферических однородных пространств, Матем. заметки, 90:5 (2011), 703-711.

[6] D. I. Panyushev, Complexity and rank of homogeneous spaces, Geom. Dedicata, 34:3 (1990), 249-269.

[7] Р. С. Авдеев, О разрешимых сферических подгруппах полупростых алгебраических групn, Труды МMO, 72:1 (2011), 5-62.

[8] В. Л. Попов, Группь Пикара однородных пространств линейных алгебраических групп и одномерные однородные векторные расслоения, Изв. АН СССР. Сер. матем., 38:2 (1974), 294-322.

[9] D. A. Timashev, Homogeneous Spaces and Equivariant Embeddings, Encyclopaedia of Mathematical Sciences, vol. 138, Springer-Verlag, Berlin-Heidelberg, 2011.

[10] D. I. Panyushev, Complexity and nilpotent orbits, Manuscripta Math., 83 (1994), 223237.

[11] P.-L. Montagard, Une nouvelle propriété de stabilité du pléthysme, Comm. Math. Helvetici, 71:3 (1996), 475-505.

[12] Э. Б. Винберг, В. Л. Попов, Теория инвариантов, в кн.: Итоги науки и техники. Современные проблемы математики. Фундаментальные направления, т. 55, ВИНИТИ, М., 1989, 137-309.

Московский государственный университет,

Поступило в редакцию механико-математический факультет

17 декабря 2010 г.

e-mail: suselr@yandex.ru

Московский государственный университет, механико-математический факультет

e-mail: nataly.gorfinkel@gmail.com 